\title{
Closed-Loop Evaluation of An Integrated Failure Identification And Fault Tolerant Control System for A Transport Aircraft
}

\author{
Jong-Yeob Shin* \\ National Institute of Aerospace, Hampton, VA 23666 \\ Christine Belcastro ${ }^{\dagger}$ and Thuan Khong $\ddagger$ \\ NASA Langley Research Center, Hampton, VA 23681
}

\begin{abstract}
Formal robustness analysis of aircraft control upset prevention and recovery systems could play an important role in their validation and ultimate certification. Such systems developed for failure detection, identification, and reconfiguration, as well as upset recovery, need to be evaluated over broad regions of the flight envelope or under extreme flight conditions, and should include various sources of uncertainty. To apply formal robustness analysis, formulation of linear fractional transformation (LFT) models of complex parameter-dependent systems is required, which represent system uncertainty due to parameter uncertainty and actuator faults. This paper describes a detailed LFT model formulation procedure from the nonlinear model of a transport aircraft by using a preliminary LFT modeling software tool developed at the NASA Langley Research Center, which utilizes a matrix-based computational approach. The closed-loop system is evaluated over the entire flight envelope based on the generated LFT model which can cover nonlinear dynamics. The robustness analysis results of the closed-loop fault tolerant control system of a transport aircraft are presented. A reliable flight envelope (safe flight regime) is also calculated from the robust performance analysis results, over which the closed-loop system can achieve the desired performance of command tracking and failure detection.
\end{abstract}

\footnotetext{
*Senior Staff Scientist, Robust and Adaptive Control, AIAA Member, email:shinjy@nianet.org.

${ }^{\dagger}$ Senior Researcher, Dynamic Systems and Control Branch, AIAA Member.

‡Researcher, Dynamic Systems and Control Branch, AIAA Member.
} 


\title{
Nomenclature
}

\author{
$\alpha, V$ : Angle of attack (rad), True airspeed, (m/sec). \\ $q, \theta$ : Pitch angle rate ( $\mathrm{rad} / \mathrm{sec})$, Pitch angle (rad). \\ $\bar{q}, \gamma \quad$ : Dynamic pressure $\left(\mathrm{N} / m^{2}\right)$, Flight path angle (rad). \\ $\delta_{e}, \delta_{s}:$ Elevator deflection (deg), Stabilizer deflection (rad). \\ $T, m$ : Thrust (N), Total mass (kg). \\ $\bar{c}, c_{7} \quad$ : Mean chord length $(\mathrm{m})$, Inertia coefficient, $1 / I_{y y}\left(\mathrm{~kg}^{-1} \mathrm{~m}^{-2}\right)$.

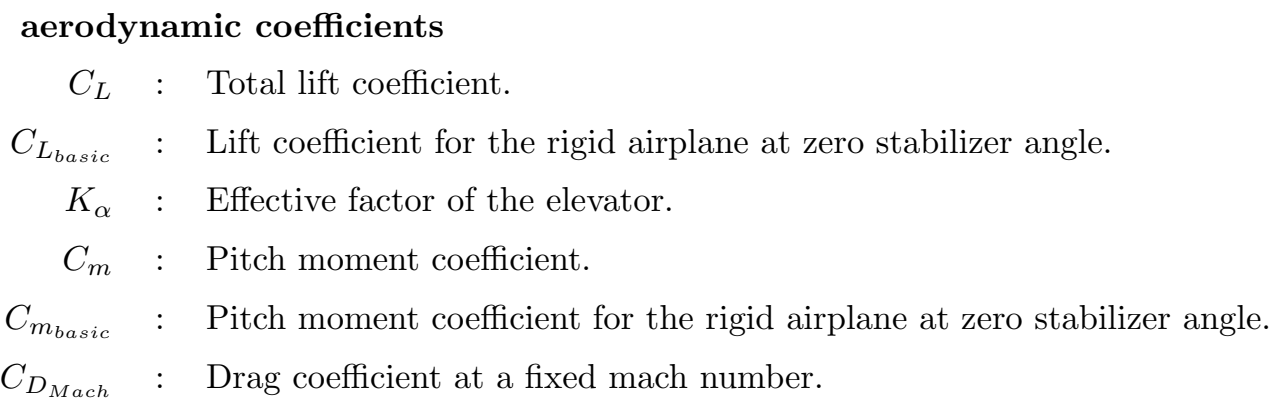

\section{Introduction}

Aircraft loss-of-control accidents [1] comprise the largest and most fatal aircraft accident category across all civil transport classes, and can result from a large array of causal and contributing factors (e.g., system and component failures, control system impairment or damage, inclement weather, inappropriate pilot inputs, etc.) occurring either individually or in combination. Research [2-5] into the characterization of the aircraft loss-of-control phenomenon as well as loss-of-control prevention and recovery system technologies is being conducted by NASA as part of its Aviation Safety Program (AvSP). In Ref. [6], it is shown that loss-ofcontrol events can involve flight beyond normal operating conditions. Moreover, these conditions are not well modeled in current transport simulations. Validation of both the mathematical models and the systems technologies for loss-of-control conditions is therefore highly nontrivial.

Certification of loss-of-control prevention and recovery systems (including failure detection, identification, and reconfiguration as well as upset recovery subsystems) for an aircraft will require a comprehensive validation process (integrating analysis, simulation, and experimental methods) to ensure the safety and reliability of these systems. Robustness analysis for systems with structured uncertainty could play an important role in this process. Robustness is a key issue in the performance of failure detection and accommodation systems. Failure detection schemes can experience performance difficulties (such as false alarms) due to system uncertainties. Robustness of the control system can mask faults and failures and make the detection problem more difficult. It is fairly common for integration of failure detection and accommodation systems to be problematic if they're designed separately. Robustness analysis can also identify worst-case combinations of uncertainties, faults and failures for use in guided Monte Carlo simulation and/or experimental studies, and could provide real-time risk mitigation during high-risk flight testing. This type of testing will be conducted utilizing a dynamically scaled transport aircraft that has been developed at the NASA Langley Research 
Center as part of the Airborne Subscale Transport Aircraft Research (AirSTAR) Testbed (see Refs. [4,5]). For an aircraft, robustness to nonlinear parameter variations over the flight envelope and at extreme flight conditions must also be considered. Ref. [7] provides an excellent treatment of applying robustness analysis methods to the clearance of flight control laws, and Ref. [3] provides a robustness analysis framework for failure detection and accommodation systems. Analytical robust control methods, such as the structured singular value ( $\mu$ see Refs. $[3,7]$ ), require the formulation of a linear fractional transformation (LFT) model of the uncertain system.

Formulation of the LFT model can be extremely difficult and time consuming, especially for aircraft problems involving parametric uncertainties (see Refs. [3,7-12]). In fact, the difficulty in formulating the uncertainty model in LFT form has been a key impediment to performing robustness analysis for these systems. Ref. [1] presents a numerical matrix-based modeling method and preliminary software tool for computing LFT models from a linear parameter varying (LPV) model of the system. This paper presents practical uses of LFT models in robustness analysis of the integrated fault tolerant control (IFTC) system for a Boeing 747-100/200 aircraft.

In Ref. [13], the IFTC system of the aircraft is designed, which includes a fault tolerant control (FTC) law and fault detection and isolation (FDI) filters. In Ref. [13], the FTC law is designed as a $H_{\infty}$ control law to minimize command tracking errors under actuator fault occurrence and the FDI filter is designed based on an affine LPV model of the Boeing 747 aircraft to generate residual signals using the geometric approach [14]. Since the FDI filter is designed based on the open-loop dynamics, the closed-loop evaluation is required before implementing it. For the closed-loop evaluation over the entire flight envelope, an LFT model from the nonlinear dynamics model of the Boeing 747-100/200 aircraft [13] is developed in this paper. The variation of the nonlinear aircraft dynamics over the flight envelope are captured by the LFT model with the $\Delta$ block, which contains key parameters such as angle of attack and velocity. To apply the robustness analysis ( $\mu$-analysis) to the closed-loop evaluation, LTI FDI filters are extracted from the LPV-FDI filters using the Jacobian linearization method. This paper presents robust performance analysis of the IFTC system for the Boeing 747 aircraft and a reliable flight envelope (safe flight regime), in which the IFTC system achieves the desired performance of command tracking and failure detection.

This paper contains the following sections. In Section 2, the LFT modeling algorithm of the matrix-based computational approach is briefly summarized. In Section 3, the analysis problem for the IFTC system of the aircraft presented in Ref. [13] is described. In Section 4, the LFT model of the longitudinal motion of the transport aircraft in Ref.[13] is developed, which can cover the nonlinear dynamics over the given flight envelope. In Section 5, a robustness analysis framework and analysis results are described, and in Section 6 the results are summarized with conclusions.

\section{Numerical Parameter LFT Modeling Approach}

The matrix-based LFT modeling method presented in Refs. $[1,3]$ is briefly summarized for completeness of this paper. Suppose the class of systems is described as

$$
\left[\begin{array}{l}
\dot{x} \\
y
\end{array}\right]=\left[\begin{array}{ll}
A(\rho) & B(\rho) \\
C(\rho) & D(\rho)
\end{array}\right]\left[\begin{array}{l}
x \\
u
\end{array}\right],
$$


where $\rho \in \mathcal{R}^{m}$ and $m$ is the number of uncertain parameters. Assume that the matrices $A(\rho), B(\rho), C(\rho)$ and $D(\rho)$ are in multi-variable polynomial matrix form such as

$$
\left[\begin{array}{ll}
A(\rho) & B(\rho) \\
C(\rho) & D(\rho)
\end{array}\right]=\left[\begin{array}{cc}
A_{o} & B_{o} \\
C_{o} & D_{o}
\end{array}\right]+\sum_{i=1}^{f_{n}}\left[\begin{array}{cc}
A_{i} & B_{i} \\
C_{i} & D_{i}
\end{array}\right] f_{i}(\rho),
$$

where $f_{i}(\rho)$ is a multi-variable polynomial function and $f_{n}$ is the total number of functions.

The LFT model of the system of Eq. (1) to be solved is depicted in Fig. 1. The matrix $\Delta(\rho)$ contains the

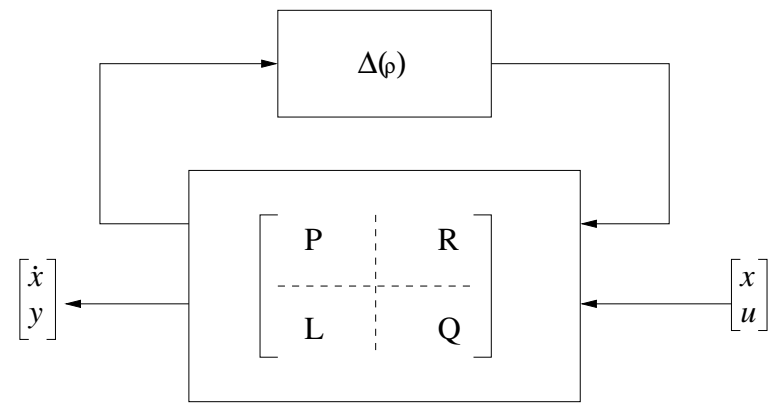

Figure 1: Block diagram of the LFT modeling problem

system uncertainties, and can be represented as follows for parametric uncertainties.

$$
\begin{gathered}
\Delta(\rho)=\operatorname{diag}\left[\delta_{1} I_{n_{1}}, \quad \delta_{2} I_{n_{2}}, \cdots, \quad \delta_{m} I_{n_{m}}\right] \\
\rho=\left[\delta_{1}, \delta_{2}, \cdots, \delta_{m}\right] \in \mathcal{R}^{m}
\end{gathered}
$$

The LFT equation associated with Fig. 1 is given below

$$
S(\rho)=L(I-\Delta P)^{-1} \Delta R+Q=S_{\Delta}(\rho)+Q .
$$

where the matrix $S(\rho)$ is a compact representation of the system model. The matrix $Q$ represents the nominal system model. The interconnection matrices $P, R$, and $L$ are to be determined for the uncertain component of $S$, such as

$$
S_{\Delta}(\rho)=L(I-\Delta P)^{-1} \Delta R .
$$

Eq. (6) can be solved for multivariate polynomial problems by replacing the matrix inversion with a finite series expansion and a nilpotency condition,

$$
\begin{gathered}
S_{\Delta}(\rho)=L \Delta R+L\left[\Delta P+(\Delta P)^{2}+\cdots+(\Delta P)^{r}\right] \Delta R \\
(\Delta P)^{r+1}=0
\end{gathered}
$$

where $r$ is determined by the degree of the largest nonzero term in $S_{\Delta}(\rho)$.

The blocks of $L$ and $R$, and the main-diagonal blocks of $P$ are solved simultaneously for each uncertain parameter $\delta_{i}$ and the off-diagonal blocks of $P$ are each solved using the appropriate cross terms of $S_{\Delta}(\rho)$. The detailed procedures are described in Refs. $[1,3]$. There are other available software tools developed by ONERA [15] and MuSyn. Inc [16], respectively. In this paper, the LFT models of the transport aircraft are obtained using the matrix-based LFT modeling method and the uncertainty model method provided in the Robust Toolbox in MATLAB, since the software tools by ONERA and MuSyn Inc. generate LFT models with the similar sized $\Delta$ block [1]. The comparison results of the LFT models are presented in subsection 4.4. 


\section{Analysis Problem on The IFTC System}

The IFTC system of the Boeing 747-100/200 aircraft presented in Ref. [13] is briefly described here to carry out detailed closed-loop evaluation of the system over the entire flight envelope. The IFTC system shown in Fig. 2 contains a fault tolerant control law, fault detection and isolation filters, actuators and sensors. In Ref. [13], the fault tolerant control law was designed as a passive fault tolerant control law minimizing

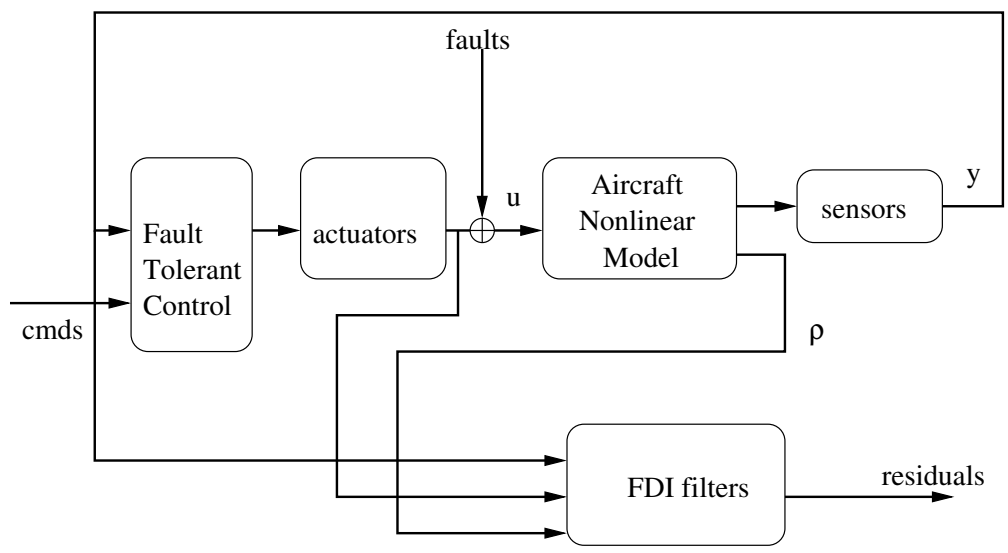

Figure 2: The simplified block diagram of the IFTC system of a transport aircraft in Ref.[13].

flight-path angle and velocity command tracking errors under fault occurrence. Actuator faults are modeled as additive signals on each control channel. In Ref. [13], the LPV-FDI filters were designed, based on the affine LPV model of the longitudinal motion of the Boeing 747-100/200 aircraft as residual generators using the geometric approach [14].

To evaluate the IFTC system over the entire flight envelope, the IFTC system is augmented with performance weighing functions $\left(W_{p}\right.$ and $\left.W_{f}\right)$, a fault scale matrix $\left(F_{s}\right)$, and ideal closed-loop dynamics $\left(T_{i}\right)$, as shown in Fig. 3. Note that the nonlinear aircraft dynamics in the augmented closed-loop system shown in Fig. 2 are replaced with the LFT model with the block $\Delta_{\text {model }}(\alpha, V)$. Recall that the LFT model with the block $\Delta_{\text {model }}$ has to capture the nonlinear dynamics over the entire flight envelope. The block $\Delta_{a c t}$ shown in Fig. 3 represents unmodeled actuator dynamics. The augmented IFTC system is converted into the generalized block diagram shown in Fig. 4 with the uncertain block $\Delta=\operatorname{diag}\left\{\Delta_{\text {model }}, \Delta_{a c t}\right\}$, which is useful for robustness analysis ( $\mu$-analysis).

For the closed-loop evaluation of the IFTC system, robust performance in an $H_{\infty}$ sense for the subsystems $\left(M_{e c}, M_{e f}, M_{f c}\right.$, and $\left.M_{f f}\right)$ shown in Fig. 4 is interpreted as follows:

1. $\mu\left(M_{e c}\right)$ : Robustness to command tracking errors in control performance over the entire flight envelope with/without the unmodeled actuator dynamics.

2. $\mu\left(M_{f f}\right)$ : Robustness to fault detection errors in FDI filter performance over the entire flight envelope with/without the actuator unmodeled dynamics.

3. $\mu\left(M_{e f}\right)$ : Effect of faults on command tracking errors over the entire flight envelope.

4. $\mu\left(M_{f c}\right)$ : Effect of commands on fault detection errors over the entire flight envelope. 


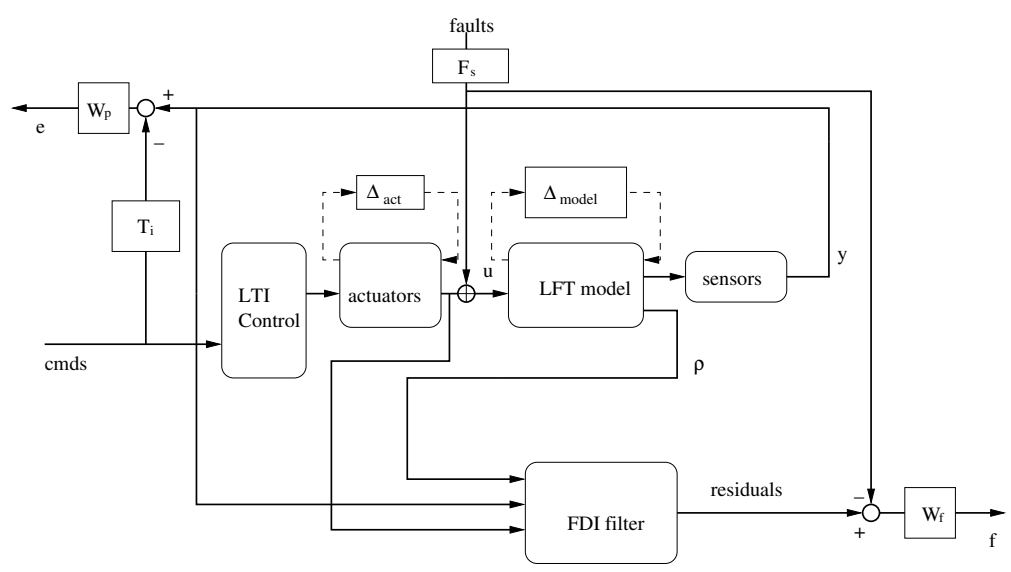

Figure 3: Augmented closed-loop interconnection block diagram of the IFTC system of a transport aircraft.

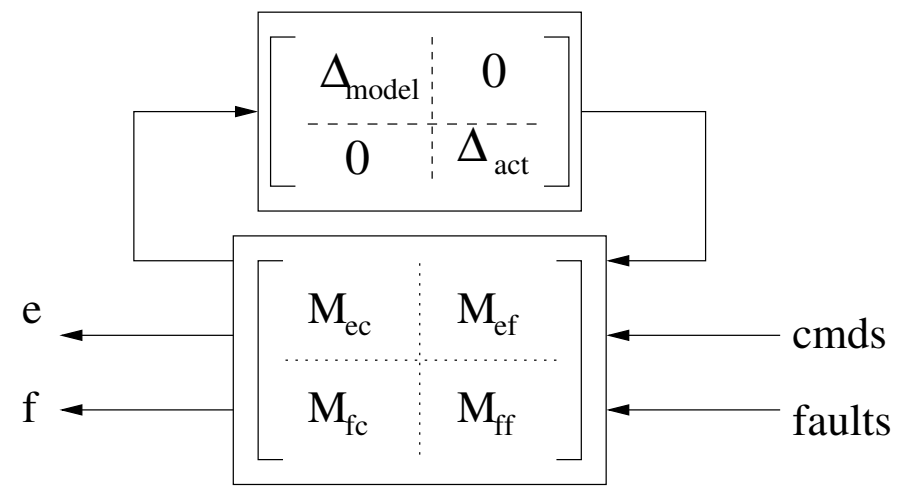

Figure 4: Generalized block diagram of the IFTC system with uncertainties.

\section{LFT Modeling of The Longitudinal Motion}

For the closed-loop evaluation over the entire flight region, it is important to develop a reliable LFT model which can represent the original nonlinear dynamics of the longitudinal motion. This section presents the detailed assumptions and procedures to reformulate the nonlinear equations of longitudinal motion into quasi-LPV form in Eq. (1). The nonlinear dynamic model of the longitudinal motion presented in Ref. [13] is summarized to develop an LPV model and an LFT model.

\subsection{High Fidelity Nonlinear Model}

The full nonlinear equations of the Boeing 747 longitudinal motion are taken from Ref. [13] over the up-and

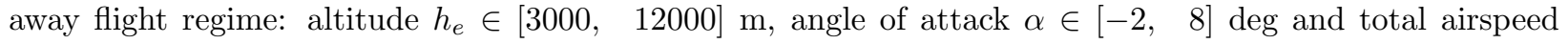
$V \in[150,280]$. In this paper, the entire flight envelope is defined as the up-and-away flight regime. The 
detailed nonlinear equations of motion are

$$
\begin{gathered}
\dot{\alpha}=\left[1-\frac{\bar{q} S \bar{c}}{2 m V^{2}}\left(1.45-1.8 x_{c g}\right) \frac{d C_{L}}{d q}\right] q+\left[-\frac{\bar{q} S}{m V} K_{\alpha} \frac{d C_{L}}{d \delta_{e}}\right] \delta_{e} \\
\quad+\left[-\frac{4}{m V}(\sin \alpha+0.0436 \cos \alpha)\right] T+\frac{1}{V}(\sin \alpha \sin \theta+\cos \alpha \cos \theta) g-\frac{\bar{q} S}{m V} C_{L_{b a s i c}}, \\
\dot{q}=\frac{c_{7} \bar{q} S \bar{c}^{2}}{2 V}\left[\frac{d C_{m}}{d q}-\frac{1}{\bar{c}}\left(1.45-1.8 x_{c . g .}\right) \frac{d C_{L}}{d_{q}}\left(\cos \alpha \bar{x}_{c . g .}+\sin \alpha \bar{z}_{c . g .}\right)\right] q \\
+c_{7} \bar{q} S \bar{c} K_{\alpha}\left[\frac{d C_{m}}{d \delta_{e}}-\frac{1}{\bar{c}} \frac{d C_{L}}{d \delta_{e}}\left(\cos \alpha \bar{c}_{c . g .}+\sin \alpha \bar{z}_{c . g .}\right)\right] \delta_{e} \\
+c_{7} \bar{q} S \bar{c} K_{\alpha} \frac{d C_{m}\left(h_{e}, M\right)}{d \delta_{s}} \delta_{s}+c_{7} S_{z e n g} T+c_{7} \bar{q} S \bar{c} C_{m_{\text {basic }}}(\alpha, M) \\
+c_{7} \bar{q} S\left[C_{D_{M a c h}}\left(M, C_{L}\right)\left(\cos \alpha \bar{z}_{c . g .}-\sin \alpha \bar{x}_{c . g .}\right)-C_{L_{b a s i c}}\left(\alpha_{w}, M\right)\left(\cos \alpha \bar{x}_{c . g .}+\sin \alpha \bar{z}_{c . g .}\right)\right] \\
\dot{V}=\frac{4}{m}(\cos \alpha-0.0436 \sin \alpha) T+(\sin \alpha \cos \theta-\cos \alpha \sin \theta) g-\frac{\bar{q} S}{m} C_{D_{M a c h}}\left(M, C_{L}\right) \\
\dot{\theta}=q \\
\dot{h}_{e}=(\cos \alpha \sin \theta-\sin \alpha \cos \theta) V
\end{gathered}
$$

Note that aerodynamic coefficients and their derivatives are calculated from the look-up tables described in Ref. [17]. Here, the thrust generated by four engines is described by " $4 \mathrm{~T}$ " using one variable and also the four elevators are described as one variable $\delta_{e}$ for simplicity. The full nonlinear motions are rewritten as an affine LPV model to generate LPV-FDI filters in Ref. [13]. The affine LPV model is described in the next section.

\subsection{Affine LPV Model of the Longitudinal Motion}

In Ref. [13], the affine LPV model is developed from the nonlinear equations of Eqs. (9)-(13) under the assumptions that each element in the nonlinear equation which has small variation over the flight envelope is considered as constant and the nine scheduling parameters are introduced to cover the nonlinear dynamics over the affine parameter space described by their vertex points. The nine scheduling parameters for the affine LPV model in Ref. [13] are

$$
\begin{gathered}
\rho_{1}=\bar{q}, \quad \rho_{2}=\bar{q} / V, \quad \rho_{3}=1 / V, \quad \rho_{4}=\gamma, \quad \rho_{5}=C_{L_{b a s i c}} \bar{q} / V \\
\rho_{6}=\frac{d C_{L}}{d \delta_{e}} \frac{\bar{q}}{V}, \quad \rho_{7}=C_{D_{M a c h}} \bar{q}, \quad \rho_{8}=\frac{d C_{m}}{d \delta_{e}} \bar{q}, \quad \rho_{9}=C_{m_{b a s i c}} \bar{q} .
\end{gathered}
$$

Using the scheduling parameters, the full nonlinear equations are rewritten as

$$
\begin{aligned}
\dot{\alpha} & =a_{\alpha_{1}} q+a_{\alpha_{2}} \rho_{6} \delta_{e}+a_{\alpha_{3}} \rho_{3} T+\left(a_{\alpha_{4}} \rho_{3}+a_{\alpha_{5}} \rho_{5}\right) \delta_{f i c} \\
\dot{q} & =\left(a_{q_{1}} \rho_{1}+a_{q_{2}} \rho_{7}\right) \alpha+a_{q_{3}} \rho_{2} q+\left(a_{q_{4}} \rho_{8}+a_{q_{5}} \rho_{1}\right) \delta_{e}+a_{q_{6}} \rho_{1} \delta_{s}+a_{q_{7}} T+\left(a_{q_{8}} \rho_{1}+a_{q_{9}} \rho_{9}\right) \delta_{f i c} \\
\dot{V} & =a_{V_{1}} T+\left(a_{V_{2}} \rho_{4}+a_{V_{3}} \rho_{7}\right) \delta_{f i c} \\
\dot{\theta} & =q \\
\dot{h}_{e} & =\rho_{4} V
\end{aligned}
$$

where the $a_{i}$ coefficients, written in Appendix A, are treated as constant parameters over the flight envelope. 
Recall that the state vector is defined as $x=\left[\begin{array}{lllll}\alpha & q & V & \theta & h_{e}\end{array}\right]^{T}$ and control is $u=\left[\begin{array}{llll}\delta_{e}, & \delta_{s}, & T, & \delta_{f i c}\end{array}\right]^{T}$ in Ref. [13]. Note that there is a fictitious input $\delta_{f i c}$ which is always defined as one in the simulations of the affine LPV model. Recall that when FDI filters are designed in Ref. [13], the LPV model should be affine to use geometric approach. In this paper, we convert the affine LPV model into a quasi-LPV model with two scheduling parameters, which is not affine. The affine LPV model of the Boeing 747 longitudinal model is rewritten as

$$
\dot{x}=A(\rho) x+B(\rho) u
$$

where

$$
A(\rho)=A_{0}+\sum_{i=1}^{9} A_{i} \rho_{i}, \quad B(\rho)=B_{0}+\sum_{i=1}^{9} B_{i} \rho_{i} .
$$

The detailed elements of $A$ and $B$ matrices are referred to Ref. [13].

The affine LPV model is developed for designing FDI filters not for analysis. The nine scheduling parameters of the affine LPV model are treated as independent parameters in Ref. [13] to construct FDI filters. It is not applicable for robustness analysis since the nine scheduling parameters are physically dependent on each other. In order to remove the dependency and the fictitious input, the affine LPV model is rewritten in the nonlinear form under the assumptions that altitude variations are small enough to be ignored for level flight.

\subsection{Low Fidelity Nonlinear Model of the Transport Aircraft}

The aerodynamic coefficients are fitted as polynomial functions of angle of attack and velocity for level flight over the flight envelope. Here, the coefficients are rewritten as

$$
\begin{aligned}
C_{D_{M a c h}} & =\kappa_{20} \alpha^{2}+\kappa_{10} \alpha+\kappa_{01} V+\kappa_{00} \\
\frac{d C L}{d \delta_{e}} & =\tau_{02} V^{2}+\tau_{01} V+\tau_{00} \\
C_{L_{\text {basic }}} & =\eta_{10} \alpha+\eta_{01} V+\eta_{00} \\
C m_{\text {basic }} & =\xi_{20} \alpha^{2}+\xi_{10} \alpha+\xi_{01} V+\xi_{00} \\
\frac{d C_{m}}{d \delta_{e}} & =\zeta_{02} V^{2}+\zeta_{01} V+\zeta_{00}
\end{aligned}
$$

where the constant coefficients are

$$
\begin{aligned}
& \kappa_{20}=3.27, \quad \kappa_{10}=3.48 \times 10^{-2}, \quad \kappa_{01}=4.45 \times 10^{-5}, \quad \kappa_{00}=9.92 \times 10^{-3}, \\
& \tau_{02}=-1.44 \times 10^{-7}, \quad \tau_{01}=4.26 \times 10^{-5}, \quad \tau_{00}=3.21 \times 10^{-3}, \\
& \eta_{10}=5.15, \quad \eta_{01}=1.21 \times 10^{-3}, \quad \eta_{00}=6.15 \times 10^{-3}, \\
& \xi_{20}=2.39, \quad \xi_{10}=-1.46, \quad \xi_{01}=-3.20 \times 10^{-4}, \quad \xi_{00}=0.12 \text {, } \\
& \zeta_{02}=4.35 \times 10^{-7}, \quad \zeta_{01}=-1.16 \times 10^{-4}, \quad \zeta_{00}=-1.76 \times 10^{-2} .
\end{aligned}
$$

Using the fitted aerodynamic coefficients, the affine LPV model is rewritten as a nonlinear function of angle of attack and velocity. 
The longitudinal motion is rewritten as

$$
\left[\begin{array}{c}
\dot{\alpha} \\
\dot{q} \\
\dot{V} \\
\dot{\theta}
\end{array}\right]=A(\alpha, V)\left[\begin{array}{c}
\alpha \\
q \\
V \\
\theta
\end{array}\right]+B(\alpha, V)\left[\begin{array}{c}
\delta_{e} \\
\delta_{s} \\
T
\end{array}\right]+\left[\begin{array}{c}
a_{\alpha_{4}} / V \\
0 \\
0 \\
0
\end{array}\right]
$$

where

$$
\begin{aligned}
& A(\alpha, V)=\left[\begin{array}{cccc}
0.5 \rho a_{\alpha_{5}} \eta_{10} V & a_{\alpha_{1}} & 0.5 \rho a_{\alpha_{5}}\left(\eta_{01} V+\eta_{00}\right) & 0 \\
0.5 \rho V\left(a_{q_{2}}+\left(a_{q_{1}}+a_{q_{9}} \xi_{20} \alpha+a_{q_{9}} \xi_{10}\right) V\right) & 0.5 \rho q_{q_{3}} V & 0.5 \rho\left(a_{q_{8}}+a_{q_{9}} V \xi_{01}+a_{q_{9}} \xi_{00}\right) V & 0 \\
-a_{V_{2}}+0.5 \rho V^{2} a_{V_{3}}\left(\kappa_{20} \alpha+\kappa_{10}\right) & 0 & 0.5 \rho a_{V_{3}} V\left(\kappa_{01} V+\kappa_{00}\right) & a_{V_{2}} \\
0 & 1 & 0 & 0
\end{array}\right] \\
& B(\alpha, V)=\left[\begin{array}{ccc}
0.5 \rho a_{\alpha_{2}}\left(\tau_{02} V^{3}+\tau_{01} V^{2}+\tau_{00} V\right) & 0 & a_{\alpha_{3}} / V \\
0.5 \rho\left(a_{q_{4}}\left(\zeta_{02} V^{2}+\zeta_{01} V+\zeta_{00}\right) V^{2}+a_{q_{5}} V^{2}\right) & 0.5 \rho a_{q_{6}} V^{2} & a_{q_{7}} \\
0 & 0 & a_{V_{1}} \\
0 & 0 & 0
\end{array}\right]\left[\begin{array}{c}
\delta_{e} \\
\delta_{s} \\
T
\end{array}\right] .
\end{aligned}
$$

It is observed that the system matrices of Eq. (25) are a function of only two parameters: angle of attack and velocity. Note that Eq. (25) is not in quasi-LPV form because of the term $a_{\alpha_{4}} / V$.

The dynamic model of Eq. (25) is called a low-fidelity nonlinear model, hereafter. For comparison with the high fidelity nonlinear model (hnl) of Section 4.1 and the low fidelity nonlinear model (lnl) developed here, the elevator doublet inputs are simulated. The difference comes from the fitted aerodynamic coefficients. Recall that the low fidelity nonlinear model contains polynomial fitted aerodynamic coefficients and in the high fidelity nonlinear model, aerodynamic coefficients are evaluated via linear interpolation from look-up tables in Ref. [13]. It is observed from Fig. 5 that the short period motions of the two models for $\mathrm{t}<50$ $\mathrm{sec}$ are identical to each other and the phugoid motions $(\mathrm{t}>50 \mathrm{sec})$ have some difference in time responses between two models. Hereafter, the low fidelity nonlinear motion is used to generate an LFT model for the Boeing 747 longitudinal motion.

\subsection{Quasi-LPV Model of The Aircraft}

The quasi-LPV model of the low fidelity nonlinear model in Eq. (25) is developed using the function substitution method [17-20] to convert the term $a_{\alpha_{4}} / V$ into quasi-LPV form. The function substitution method has been used to reformulate nonlinear equations into quasi-LPV form $[17,18]$. The benefit of the function substitution method is that the generated LPV model can represent the nonlinear dynamics without linear approximation (such as Jacobian linearization) over the entire possible flight region (not like state transformation limitation [18]).

To apply the function substitution method, states of the model are defined as the deviation from a reference point such as

$$
\tilde{\alpha}=\alpha-\alpha_{t}, \quad \tilde{V}=V-V_{t}, \quad \tilde{\theta}=\theta-\theta_{t},
$$



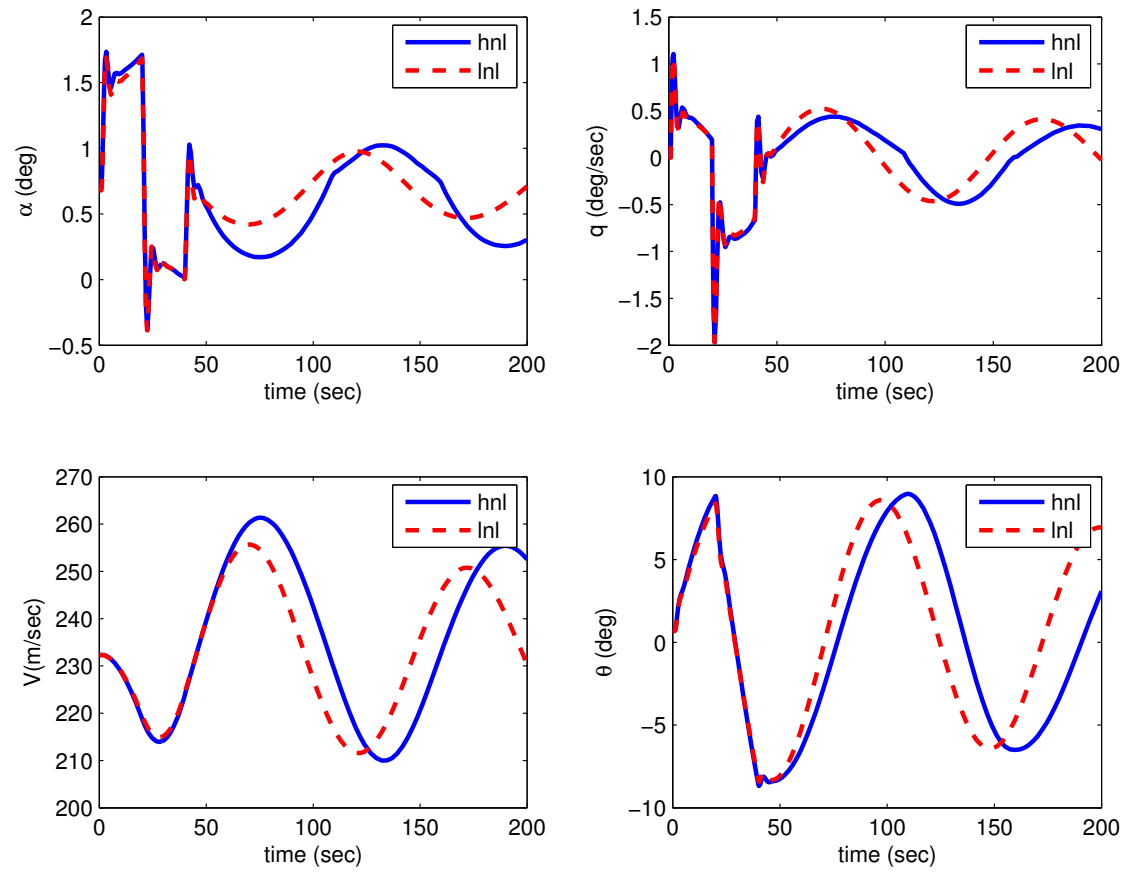

Figure 5: Time responses with elevator inputs for high and low fidelity nonlinear dynamics

where a reference point is chosen as a trim point: $\left(\alpha_{t}, 0, V_{t}, \theta_{t}\right)$. Note that $\alpha_{t}=\theta_{t}$ for level flight under a trim condition. Using Eq. (28), Eq.(25) is rewritten as

$$
\begin{aligned}
\dot{\tilde{x}} & =A(\alpha, V)\left(x_{t}+\tilde{x}\right)+B(\alpha, V)\left(u_{t}+\tilde{u}\right)+\left[\begin{array}{llll}
a_{\alpha_{4}} / V & 0 & 0 & 0
\end{array}\right]^{T}, \\
& =A(\alpha, V) \tilde{x}+B(\alpha, V) \tilde{u}+h(\alpha, V),
\end{aligned}
$$

where

$$
h(\alpha, V)=A(\alpha, V) x_{t}+B(\alpha, V) u_{t}+\left[\begin{array}{llll}
a_{\alpha_{4}} / V & 0 & 0 & 0
\end{array}\right]^{T} .
$$

Note that the control $\tilde{u}$ is $\left[\delta_{e}-\delta_{e_{t}} \delta_{s}-\delta_{s_{t}} T-T_{t}\right]^{T}$ and the trim value $u_{t}$ is $\left[\delta_{e_{t}}, \delta_{s_{t}}, T_{t}\right]^{T}$. In this paper, the trim condition is set as $\left.\left[\alpha_{t}, q_{t}, V_{t}, \theta_{t}, h_{e}\right]=\left[\begin{array}{llll}0.7(d e g), & 0, & 232(m / s e c\end{array}\right), \quad 0.7(\operatorname{deg}), \quad 7000(m)\right]$, and $\left[\delta_{e_{t}}, \delta_{a_{t}}, T_{t}\right]=[2(\operatorname{deg}), \quad-0.06(\operatorname{deg}), \quad 42991(N)]$.

Define $h(\alpha, V)=\left[\begin{array}{llll}h_{\alpha} & h_{q} & h_{V} & h_{\theta}\end{array}\right]^{T}$. Note that $h(\alpha, V)$ can be explicitly written as function of $\alpha$ and $V$. For example, the first element $h_{\alpha}$ of $h(\alpha, V)$ is given by

$$
h_{\alpha}=0.5 \rho\left(a_{\alpha_{5}} \eta_{10} \alpha_{t} V+a_{\alpha_{5}}\left(\eta_{01}+\eta_{00}\right) V_{t}+a_{\alpha_{2}}\left(\tau_{02} V^{2}+\tau_{01} V+\tau_{00}\right) V \delta_{e_{t}}\right)+\left(a_{\alpha_{3}} T_{t}+a_{\alpha_{4}}\right) / V .
$$

Using the approximation $1 / V \approx \frac{1}{V_{t}}\left(1-\tilde{V} / V_{t}\right)$ and substituting $V=V_{t}+\tilde{V}$, Eq. (31) is rewritten as

$$
h_{\alpha}=\left(l_{1}-l_{r} / V_{t}^{2}+2 l_{2} V_{t}+3 l_{3} V_{t}^{2}+\left(l_{2}+3 l_{3} V_{t}\right)\left(V-V_{t}\right)+3 l_{3}\left(V-V_{t}\right)^{2}\right) \tilde{V}
$$

where $l_{1}, l_{2}, l_{3}$, and $l_{r}$ are shown in Appendix B. After some algebraic manipulations, Eq. (30) is rewritten 
as

$$
h(\alpha, V)=\left[\begin{array}{c}
h_{\alpha} \\
h_{q} \\
h_{V} \\
h_{\theta}
\end{array}\right]=\left[\begin{array}{cc}
0 & h_{\alpha_{V}} \\
h_{q_{\alpha}} & h_{q_{V}} \\
h_{V_{\alpha}} & h_{V_{V}} \\
0 & 0
\end{array}\right]\left[\begin{array}{c}
\tilde{\alpha} \\
\tilde{V}
\end{array}\right]
$$

where $h_{\alpha_{V}}, h_{q_{\alpha}}, h_{q_{V}}, h_{V_{\alpha}}$, and $h_{V_{V}}$ are written in Appendix B. Thus, the quasi-LPV model of the transport aircraft in Ref.[13] is

$$
\dot{\tilde{x}}=\{A(\alpha, V)+H(V)\} \tilde{x}+B(\alpha, V) \tilde{u}
$$

where

$$
H(V)=\left[\begin{array}{cccc}
0 & 0 & h_{\alpha_{V}} & 0 \\
h_{q_{\alpha}} & 0 & h_{q_{V}} & 0 \\
h_{V_{\alpha}} & 0 & h_{V_{V}} & 0 \\
0 & 0 & 0 & 0
\end{array}\right] .
$$

The quasi-LPV model with the two scheduling parameters $(\alpha, V)$ can be rewritten as

$$
\dot{\tilde{x}}=\tilde{A} \tilde{x}+B \tilde{u}=\left(\sum_{i=0, j=0}\left(A_{i, j}+H_{i, j}\right) \alpha^{i} V^{j}\right) \tilde{x}+\left(B_{r} / V+\sum_{i=0, j=0} B_{i, j} \alpha^{i} V^{j}\right) \tilde{u}
$$

Component matrices $A_{i, j}, B_{i, j}$ and $H_{i, j}$ are written in Appendix B. Note that this quasi-LPV model, Eq. (36), has no fictitious input.

The $\tilde{V} \tilde{\alpha}$ and $\tilde{V}^{2} \tilde{\alpha}$ terms appear in $h_{q}$ and $h_{V}$, respectively. To decompose the terms into quasi-LPV form, we introduce parameters $p_{1}$ and $p_{2}$ such that

$$
\begin{gathered}
\tilde{V} \tilde{\alpha}=\left[p_{1}\left(V-V_{t}\right)\left(1-p_{1}\right)\left(\alpha-\alpha_{t}\right)\right]\left[\begin{array}{c}
\tilde{\alpha} \\
\tilde{V}
\end{array}\right] \\
\tilde{V}^{2} \tilde{\alpha}=\left[p_{2}\left(V-V_{t}\right)^{2}\left(1-p_{2}\right)\left(\alpha-\alpha_{t}\right)\left(V-V_{t}\right)\right]\left[\begin{array}{c}
\tilde{\alpha} \\
\tilde{V}
\end{array}\right]
\end{gathered}
$$

where

$$
0 \leq p_{1} \leq 1, \quad 0 \leq p_{2} \leq 1
$$

The values of $p_{1}$ and $p_{2}$ are chosen as one, respectively, for the quasi-LPV model to be stable at each fixed parameter value. Note that there are different sets of possible $p_{1}$ and $p_{2}$ values which make a quasi-LPV model stable. The effect of $p_{1}$ and $p_{2}$ on LFT models is still unknown and is out of this paper's scope.

For comparison between the quasi-LPV model and the low fidelity nonlinear model of Eq. (25), time responses are simulated with the elevator input. The quasi-LPV model should exactly represent the low fidelity nonlinear model since the quasi-LPV model is developed by only algebraic manipulations without any approximation except $1 / V \approx \frac{1}{V_{t}}\left(1-\tilde{V} / V_{t}\right)$. It is observed from Fig. 6 that the time responses of both the LPV model with the scheduling parameters $(\alpha, V)$ and the low fidelity nonlinear model are identical. The developed models of the Boeing 747 longitudinal motion are briefly summarized in Table 1. 

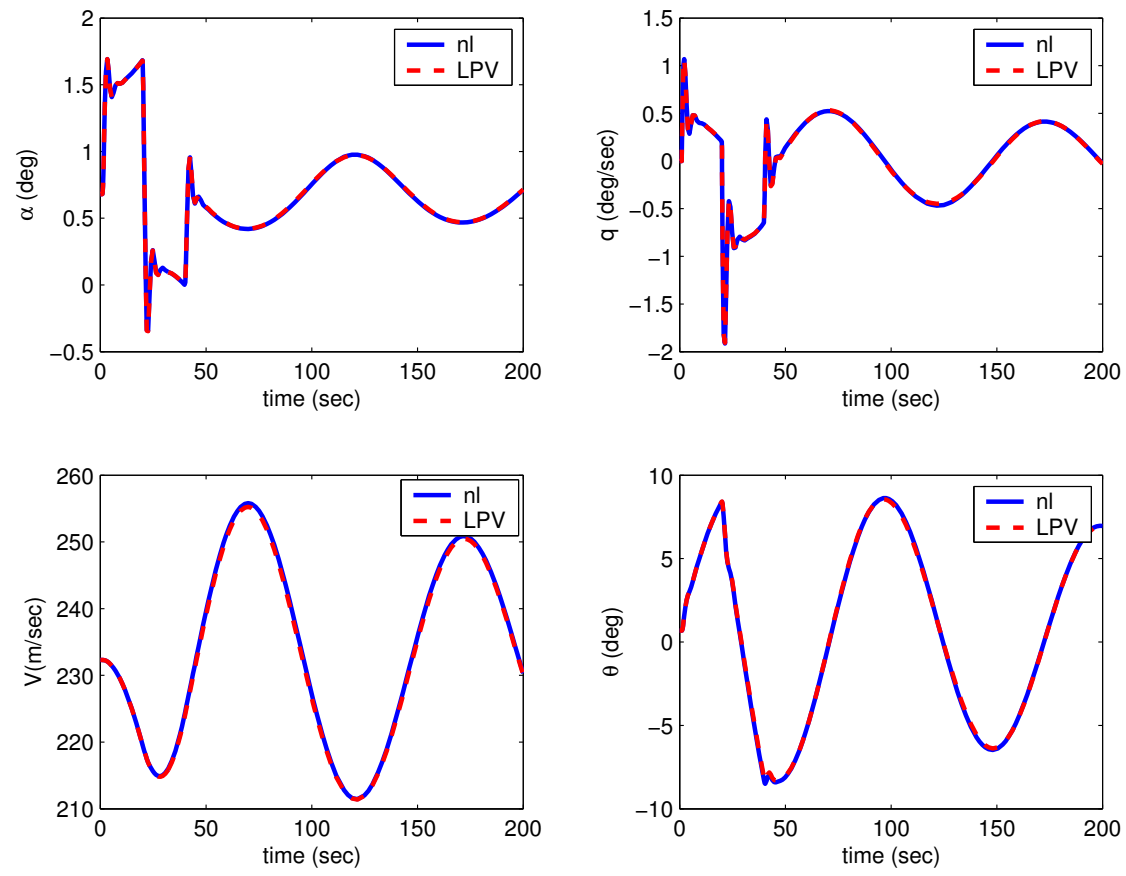

Figure 6: Time responses with elevator inputs

\subsection{LFT Models of the Boeing 747-100/200 Aircraft}

Two LFT models are obtained from the quasi-LPV model in Eq. (34) using the numerical matrix-based LFT model tool (NT) [1] and the Robust toolbox (RT) in MATLAB, respectively. The LFT model generated by $\mathrm{NT}$ has the block $\Delta_{\text {model }}=\operatorname{diag}\left\{\delta_{v} I_{6 \times 6}, \quad \delta_{\alpha}\right\}$ and the LFT model generated by RT has the block $\Delta_{\text {model }}=\operatorname{diag}\left\{\delta_{v} I_{5 \times 5}, \quad \delta_{\alpha}\right\}$. Recall that it is demonstrated in Ref. [1] that the two software tools can generate reliable LFT models which can represent the original nonlinear dynamics. In this paper, open-loop time simulations of two developed LFT models is omitted for the limited space. For comparison, the robust analysis results of the closed-loop system with each LFT model are presented in Section 5.

In order to confirm that the developed LFT model is useful for the closed-loop evaluation of the Boeing 747-100/200 aircraft, the closed-loop system is simulated with the LFT model instead of the nonlinear aircraft model (shown in Fig.2). The $\gamma$ command used in the simulation is a square wave with 3 deg amplitude, starting at $20 \mathrm{~s}$ and ending at $95 \mathrm{~s}$ and the velocity command is a step signal starting at $25 \mathrm{~s}$ as seen in Fig. 7. The fault scenario applied in elevator and the throttle channel can be seen in residual plots of Fig. 8. Based on the simulation results shown in Figs. 7 and 8, the nonlinear aircraft model can be replaced with the developed LFT model. Hereafter, the developed LFT model is used for the IFTC closed-loop evaluation over the entire flight envelope. 
Table 1: Summary of Models of the Boeing 747 longitudinal motion

\begin{tabular}{|c|c|}
\hline Model & Comment \\
\hline High fidelity model [13] & $\begin{array}{l}\text { - Full nonlinear equation of motion using aerodynamic coefficients eval- } \\
\text { uated from the look-up tables. }\end{array}$ \\
\hline Affine LPV model [13] & $\begin{array}{l}\text { - Assume that nine scheduling parameters are independent. } \\
\text { - Assume small variations of parameters }\left(a_{i}\right) \text { are constant over the flight } \\
\text { envelope. } \\
\text { - The fictitious input is set as one. }\end{array}$ \\
\hline Low fidelity model & $\begin{array}{l}\text { - Polynomial fitted aerodynamic coefficients are used. } \\
\text { - Assume the parameters } a_{i} \text { are constant. } \\
\text { - The fictitious input is not in the model. }\end{array}$ \\
\hline Quasi-LPV model & $\begin{array}{l}\text { - Accurate representation of the low fidelity nonlinear model under the } \\
\text { approximation } 1 / V=\frac{1}{V_{t}}\left(1-\tilde{V} / V_{t}\right) \text {. } \\
\text { - There are only two scheduling parameters }(\alpha, V) \text {. }\end{array}$ \\
\hline LFT model & $\begin{array}{l}\text { - Exact representation of the quasi-LPV model. } \\
\text { - Using the numerical matrix-based LFT modeling tool or the Robust } \\
\text { toolbox in MATLAB. }\end{array}$ \\
\hline
\end{tabular}



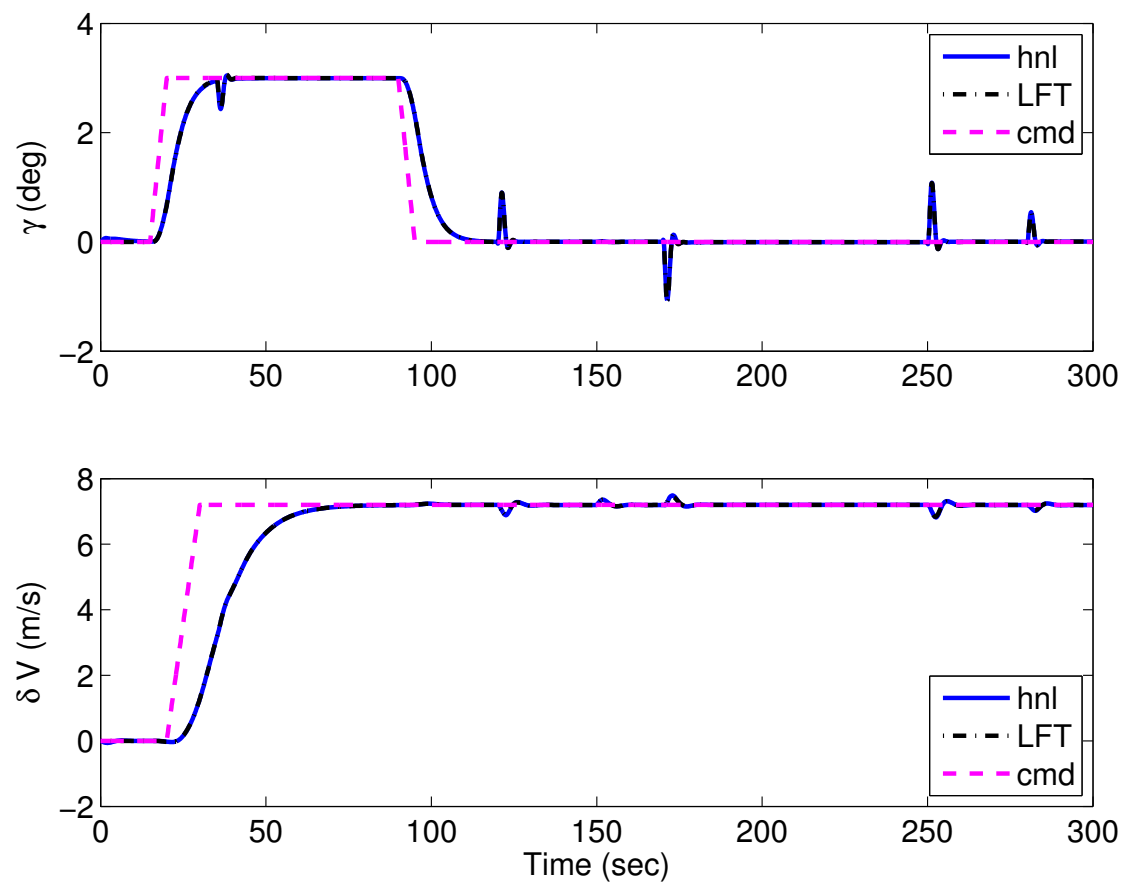

Figure 7: $\gamma$ and $V$ command time responses with high fidelity nonlinear (hnl) and the LFT models.
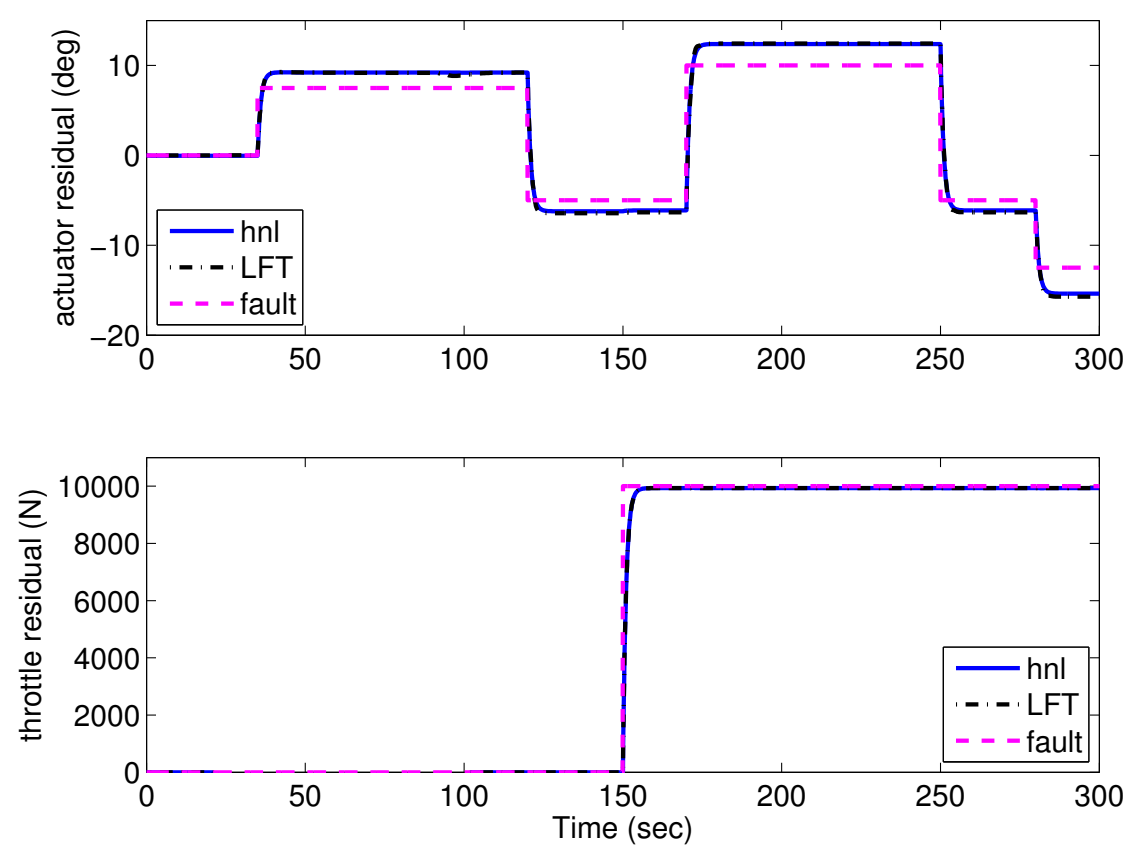

Figure 8: Fault simulations with high fidelity nonlinear (hnl) and LFT models. 


\section{$5 \quad$ Analysis Results}

The closed-loop evaluation of the Boeing 747 aircraft defined in Section 3 is formulated into generalized $\mu$ problems shown in Fig. 4. Recall that the robust performance analysis is represented by the upper bounds of $\mu$ values of each subsystem such as $M_{e c}$ : robust command tracking control performance, $M_{e f}$ : effect of commands on fault detection errors, $M_{f c}$ : effect of faults on command tracking error, and $M_{f f}$ : robust fault detection errors with/without the unmodeled actuator dynamics.

The command tracking performance weighting function $W_{p}$ is defined as $\operatorname{diag}\left(\frac{40(s / 100+1)}{s / 0.005+1}, \frac{100(s / 100+1)}{s / 0.005+1}\right)$ in this analysis to allow the flight path angle and the velocity to track their commands in the $2.5 \%$ and $1 \%$ error ranges, respectively, over the low frequency region. The fault scale matrix $F_{s}$ shown in Fig. 3 is set as $\operatorname{diag}(10(\mathrm{deg}), 10000 \mathrm{~N})$ to represent $10 \mathrm{deg}$ and $10000 \mathrm{~N}$ faults in the elevator and the throttle channels, respectively. The fault detection weighting function $W_{f}$ is chosen as $\operatorname{diag}\left(\frac{0.35(s / 1000+1)^{2}}{(s / 0.5+1)^{2}}, \frac{0.002(s / 1000+1)^{2}}{(s / 0.1+1)^{2}}\right)$ which represents about $3 \mathrm{deg}$ and $500 \mathrm{~N}$ detection errors for 10 deg elevator fault and $10000 \mathrm{~N}$ throttle fault, respectively, over the low frequency range. The block $T_{i}$ in Fig. 3 is for the ideal transfer function of $\gamma$ and $V$ tracking. The block $T_{i}=\operatorname{diag}\left(\frac{0.35^{2}}{s^{2}+0.7 s+0.35^{2}}, \frac{0.15^{2}}{s^{2}+0.3 s+0.15^{2}}\right)$ taken from Ref. [13] was used for designing the $H_{\infty}$ control law as the fault tolerant control shown in Fig. 3. The actuator models [13] are $\frac{37}{s+37}$ for the elevator actuator and $\frac{0.5}{s+0.5}$ for the throttle actuator. Here, the unmodeled actuator dynamics are defined as $\operatorname{diag}\left(\frac{0.1(s / 10+1)}{s / 500+1}, \frac{0.1(s / 10+1)}{s / 500+1}\right)$ to represent $10 \%$ unmodeled dynamics over the low frequency range $(<10 \mathrm{r} / \mathrm{s})$ and $500 \%$ over the high frequency range $(>500 \mathrm{r} / \mathrm{s})$. The uncertainty associated with the unmodeled dynamics is defined as a complex number, $\Delta_{a c t}=\operatorname{diag}\left(\delta_{1}, \delta_{2}\right), \delta_{1,2} \in \mathcal{C}$. The sensor model is approximated as an ideal sensor for consistency with Ref. [13].

The $\mu$ upper bounds of each subsystem with/without unmodeled actuator dynamics are calculated using the Robust Toolbox and are plotted in Fig. 9. It is observed from Fig. 9 that the effect of the unmodeled dynamics on the $\mu$ upper bounds is minor for each subsystem. The left top plots of Fig. 9 show that small performance degradation of command tracking is expected over the entire flight envelope since the maximum values of $\bar{\mu}$ over the frequency range is about 1.3 without the $\Delta_{\text {act }}$ block. Recall that the uncertain block $\Delta_{\text {model }}$ is a function of $\alpha$ and $V$ and contains the variations of the nonlinear dynamics over the flight envelope.

The $\mu$ upper bounds of $M_{e f}$ shown on the right top plots of Fig. 9 represent the robustness of fault effect on the command tracking error. The plots imply that faults do not affect on command tracking in this IFTC system. According to the top plots (left and right) in Fig. 9, it is observed that the designed $H_{\infty}$ control law can robustly achieve the desired command tracking performance with small degradation under fault occurrence over the entire flight envelope.

The $\mu$ upper bounds of $M_{f f}$ shown on the right bottom plots of Fig. 9 represent the robustness of fault detection errors by the FDI filters. The plots imply that the FDI filter can detect 10 deg elevator and 10000 $\mathrm{N}$ throttle faults in the error range defined by $W_{f}$ over the flight envelope with zero commands $\left(\gamma_{c m d}=0\right.$, $\left.V_{c m d}=0\right)$. The $\mu$ upper bounds of $M_{f c}$ shown on the left bottom plots of Fig.9 represent the robustness of command effect on the fault detection errors. The plots imply that the command signals can affect fault detection errors over the flight envelope. Recall that the $\gamma$ and $V$ command sizes are $15 \mathrm{deg}$ and $10 \mathrm{~m} / \mathrm{sec}$, respectively. For comparison, we set $\gamma$ and $V$ command sizes: 1) $1 \mathrm{deg} \gamma$ and $1 \mathrm{~m} / \mathrm{sec}$ velocity commands $(1 \mathrm{~g} 1 \mathrm{~V}), 2) 2 \operatorname{deg} \gamma$ and $2 \mathrm{~m} / \mathrm{sec}$ velocity commands $(2 \mathrm{~g} 2 \mathrm{~V}), 3) 5 \mathrm{deg} \gamma$ and $5 \mathrm{~m} / \mathrm{sec}$ velocity commands $(5 \mathrm{~g} 5 \mathrm{~V})$ and 4) $15 \operatorname{deg} \gamma$ and $10 \mathrm{~m} / \mathrm{sec}$ velocity commands $(15 \mathrm{~g} 10 \mathrm{~V})$. The $\mu$ upper bounds for each case are calculated and are shown in Fig. 10. It is observed from Fig. 10 that smaller $\gamma$ and $V$ commands decrease the $\mu$ upper bounds. From the bottom plots (right and left) of Fig 9 and Fig. 10, it is noticed that the 

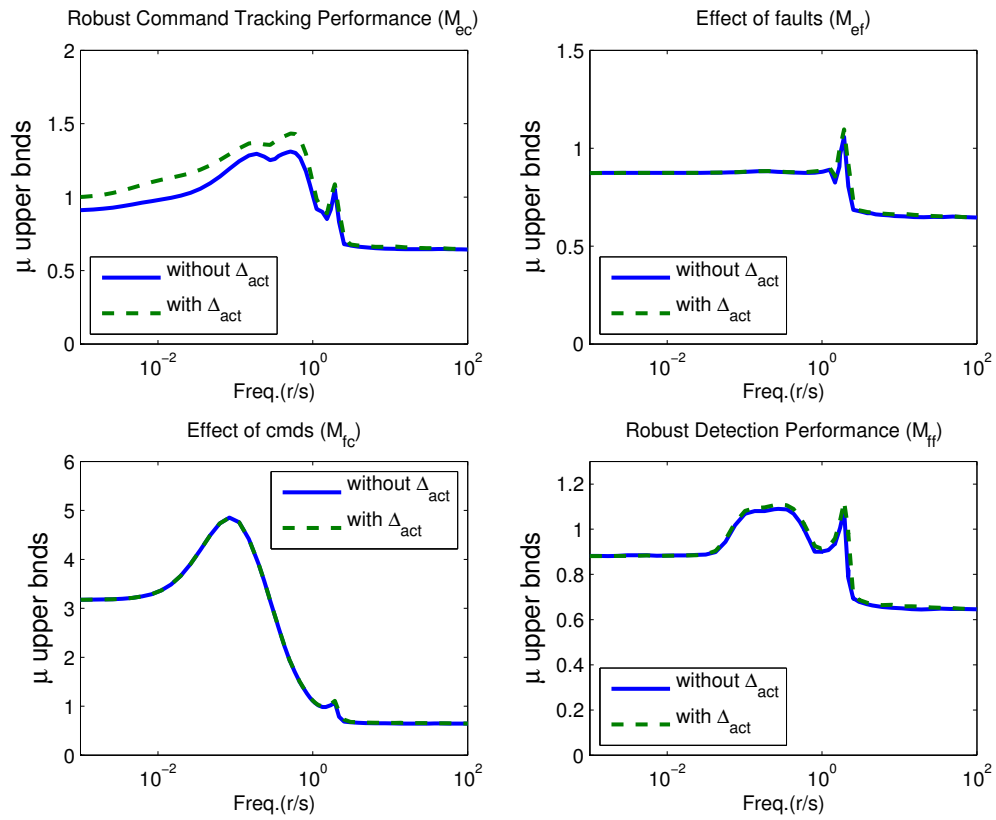

Figure 9: The upper bounds of $\mu$ values for each subsystem with/without the unmodeled actuator dynamics.

LTI-FDI filter can robustly detect faults under the condition that $\gamma$ and $V$ command sizes are small. Recall that the FDI filters designed in Ref. [13] are the LPV filters not LTI filters. The LPV filters may have more robustness and less coupling effects of the commands since the filter gains can be adjusted as angle of attack and velocity change.

Now, we can calculate a flight regime in which each subsystem can robustly achieve the desired performance under fault occurrence. This flight region, called a reliable flight envelope $(\mathcal{E})$, is calculated as

$$
\mathcal{E}(\alpha, V)=\left\{(\alpha, V) \mid\left\|\Delta_{\text {model }}(\alpha, V)\right\|_{\infty}<\frac{1}{\max _{\omega} \bar{\mu}(\omega)}\right\}
$$

where $\bar{\mu}$ is the upper bound of $\mu$ and $\omega$ is frequency. Each reliable flight envelope is shown in Fig. 11. The cross symbol in Fig. 11 is the reference point (trim point) for the LFT model. 


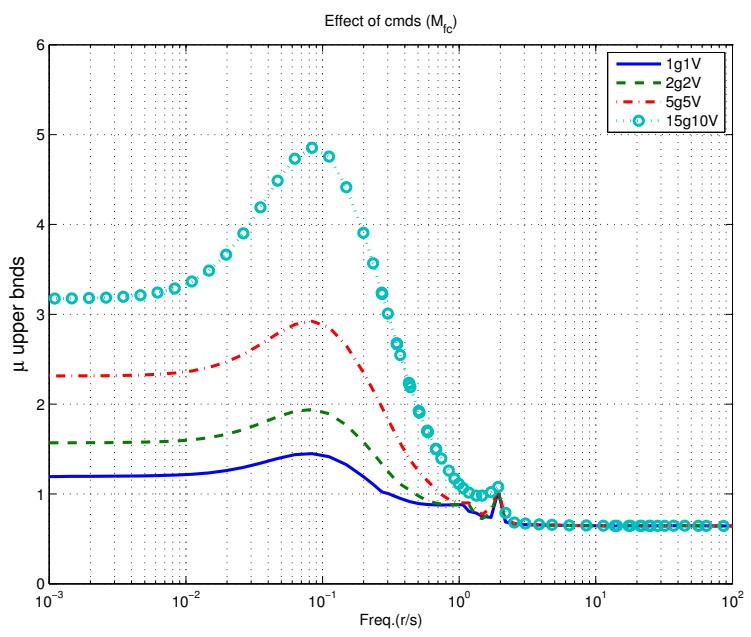

Figure 10: The $\mu$ upper bounds of $M_{f c}$ with different sizes of $\gamma$ and $V$ commands.

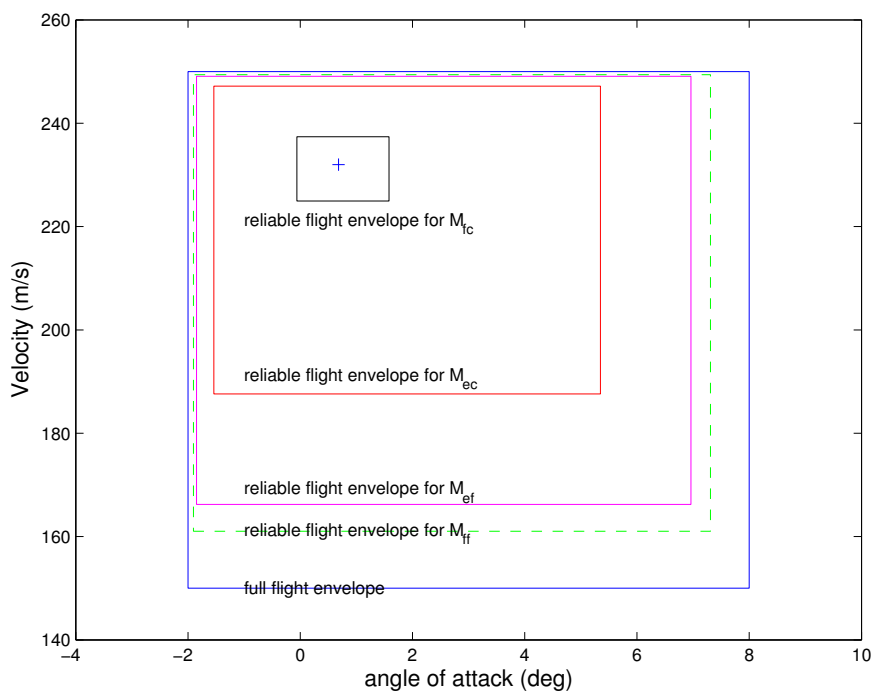

Figure 11: The reliable flight regime for each subsystem. 
Recall that the calculated reliable flight regime is dependent on the pre-defined weighting functions $W_{p}$, $W_{f}, F_{s}$ and $T_{i}$. When performance weighting functions are relaxed, the size of reliable flight regimes increase.
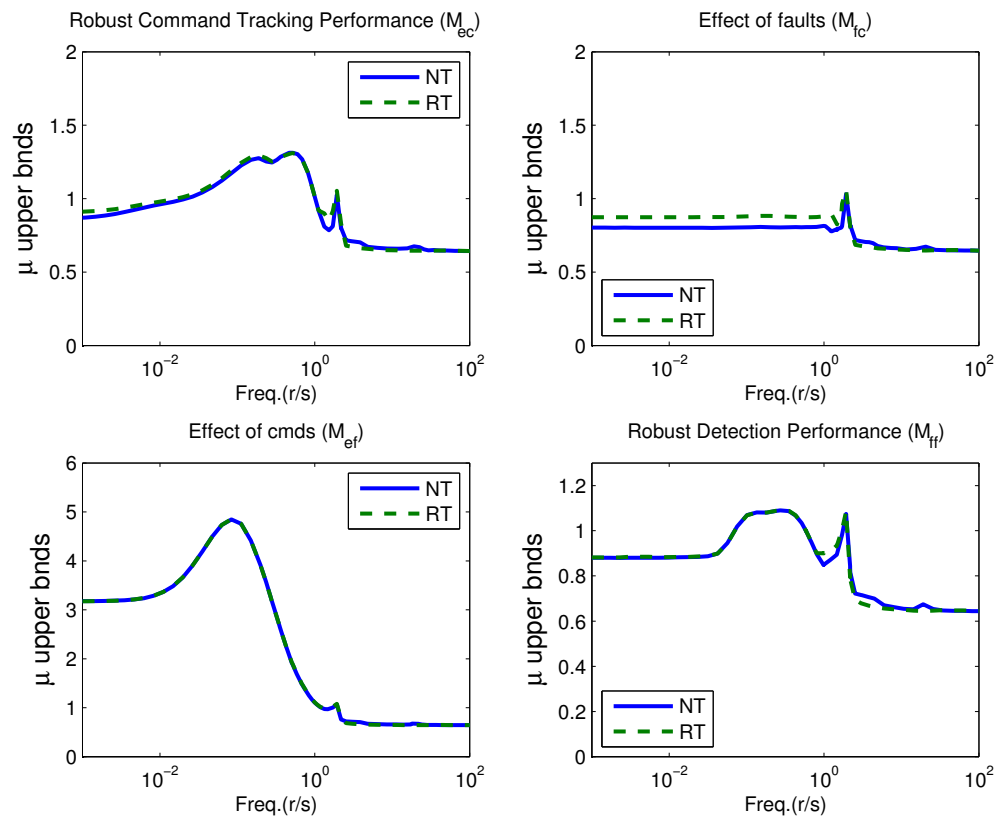

Figure 12: The $\mu$ upper bound values of subsystems based on LFT models developed by the NASA Tool (NT) and the Robust tool (RT) in MATLAB

In this paper, the two LFT models are developed using the numerical matrix-based LFT modeling tool (NT) and the Robust toolbox (RT), respectively. Fig. 12 shows the robust performance analysis results using each LFT model. It is observed that the robust performance analysis results are very similar to each other.

In this analysis framework, the LFT model of the aircraft nonlinear dynamics is generated by the function substitution method, in which a reference point is chosen as one of trim points. Generally, the LFT model is dependent on the choice of a reference point. For comparison, another LFT model is generated with a different trim point. The analysis results using the LFT model are very similar to the results shown in Fig. 9. The detailed plots are omitted for space limitation. In this analysis framework, the control law and the FDI filter are LTI systems. The IFTC system presented in Ref. [13] has an LPV-FDI filter. Recall that the LTI-FDI filter is extracted from the LPV-FDI filter using the Jacobian linearization method around the trim point. The robust analysis results of the IFTC system with the LPV FDI filter would be different from the analysis results presented in this paper because of linearization of the filter. For a further research, an IFTC system with LPV-FDI filters will be considered in robustness analysis of the closed-loop system. In the robustness analysis framework, actuator failure effects will be considered in calculating a reliable flight region. In addition, the worst flight condition for the closed-loop system will be calculated, based on actuator fault models represented as LFT models. 


\section{Conclusion}

In this paper, the closed-loop evaluation of the IFTC system is formulated into the generalized $\mu$ analysis problem using the LFT model that covers the nonlinear dynamics of the aircraft over the entire flight regime. The LFT model is developed from the parameterized system(quasi-LPV model) generated by using the function substitution method. The detailed procedure of LFT modeling of the aircraft nonlinear dynamics is presented in this paper. Using the well-developed $\mu$ analysis method, the robustness of command tracking error, command effect on fault detection errors, fault effect on command tracking error, and fault detection error are calculated as $\mu$ upper bounds over the flight envelope with/without the actuator unmodeled dynamics. Using the presented analysis framework, the IFTC system of the transport aircraft is analyzed. Based on the analysis results, the flight envelope is calculated over which the closed-loop system can achieve the desired performance of each subsystem.

\section{Appendix A}

The constant $a_{i}$ coefficients of Eq. (16) are

$$
\begin{aligned}
& a_{\alpha_{1}}=1-\frac{\bar{q} S \bar{c}}{2 m V^{2}}\left(1.45-1.8 x_{c g}\right) \frac{d C_{L}}{d q} \\
& a_{\alpha_{2}}=-(S / m) K_{\alpha} \\
& a_{\alpha_{3}}=-(4 / m)(\sin \alpha+0.0436 \cos \alpha) \\
& a_{\alpha_{4}}=(\sin \alpha \sin \theta+\cos \alpha \cos \theta) g \\
& a_{\alpha_{5}}=-(S / m) \\
a_{q_{1}}= & -c_{7} S C L_{\alpha} \bar{x}_{c . g} . \\
a_{q_{2}}= & -c_{7} S \bar{x}_{c . g} . \\
a_{q_{3}}= & 0.5 c_{7} S \bar{c}^{2}\left(\frac{d C_{m}}{d q}-\frac{1}{\bar{c}}\left(1.45-1.8 x_{c . g}\right) \frac{d C_{L}}{d q} \bar{x}_{c . g .}\right) \\
a_{q_{4}}= & c_{7} S \bar{c} K_{\alpha} \\
a_{q_{5}}= & -c_{7} S \bar{c} \bar{x}_{c . g} \cdot \frac{d C_{L}}{d \delta_{e}} \\
a_{q_{6}}= & c_{7} S \bar{c} K_{\alpha} \frac{D C_{m}}{d \delta_{s}} \\
a_{q_{7}}= & c_{7} S_{Z_{e n g}} \\
a_{q_{8}}= & -c_{7} S C_{L_{o}} \bar{x}_{c . g .} \\
a_{q_{9}}= & c_{7} S \bar{c} \\
& a_{V_{1}}=\frac{1}{m}(\cos \alpha-0.0436 \sin \alpha) 4 \\
& a_{V_{2}}=-g \\
& a_{V_{3}}=-(S / m)
\end{aligned}
$$




\section{Appendix B}

The element of $H(V)$ in Eq. (35) is as follows:

$$
\begin{aligned}
h_{\alpha_{V}} & =h_{a V_{0}}+h_{a V_{1}} V+h_{a V_{2}} V^{2} \\
h_{q_{\alpha}} & =k_{21} V^{2} \\
h_{q_{V}} & =h_{q V_{0}}+h_{q V_{1}} V+h_{q V_{2}} V^{2}+h_{q V_{3}} V^{3} \\
h_{v_{\alpha}} & =C_{21} V^{2} \\
h_{v_{v}} & =h_{V V_{0}}+h_{V V_{1}} V
\end{aligned}
$$

Each coefficient in Eq. (44) can be written as function of the trim values.

$$
\begin{aligned}
& h_{a V_{0}}=l_{1}-l r / V_{t}^{2}+l_{2} V_{t}+l_{3} V_{t}^{2} \\
& h_{a V_{1}}=l_{2}+l_{3} V_{t} \\
& h_{a V_{2}}=l_{3} \\
h_{q V_{0}} & =k_{21} V_{t} \alpha_{t}+k_{40} V_{t}^{3}+k_{30} V_{t}^{2}+k_{20} V_{t}+k_{10} \\
h_{q V_{1}} & =k_{21} \alpha_{t}+k_{40} V_{t}^{2}+k_{30} V_{t}+k_{20} \\
h_{q V_{2}} & =k_{40} V_{t}+k_{30} \\
h_{q V_{3}} & =k_{40} \\
& h_{V V_{0}}=C_{21} V_{t} \alpha_{t}+C_{20} V_{t}+C_{10} \\
& h_{V V_{1}}=C_{21} \alpha_{t}+C_{20}
\end{aligned}
$$

where

$$
\begin{gathered}
l_{r}=a_{\alpha_{3}} T_{t}+a_{\alpha_{4}} \\
l_{3}=0.5 \rho a_{\alpha_{2}} \delta_{e_{t}} \tau_{02} \\
l_{2}=0.5 \rho a_{\alpha_{2}} \delta_{e_{t}} \tau_{01} \\
l_{1}=0.5 \rho\left(a_{\alpha_{2}} \delta_{e_{t}} \tau_{00}+\left(\eta_{10} \alpha_{t}+\eta_{01} V_{t}\right)\right) \\
k_{21}=0.5 \rho a_{q_{9}} \xi_{20} \alpha_{t} \\
k_{40}=0.5 \rho a_{q_{4}} \zeta_{02} \delta_{e_{t}} \\
k_{30}=0.5 \rho a_{q_{4}} \zeta_{01} \delta_{e_{t}} \\
k_{20}=0.5 \rho\left(a_{q_{1}} \alpha_{t}+a_{q_{9}} \xi_{10} \alpha_{t}+a_{q_{9}} \xi_{01} V_{t}+a_{q_{4}} \zeta_{00} \delta_{e_{t}}+a_{q_{5}} \delta_{e_{t}}+a_{q_{6}} \delta_{s_{t}}\right) \\
k_{10}=0.5 \rho\left(a_{q_{2}} \alpha_{t}+a_{q_{8}} V_{t}+a_{q_{9}} \xi_{00} V_{t}\right) \\
C_{21}=0.5 \rho \alpha_{t} a_{V_{3}} \kappa_{20} \\
C_{20}=0.5 \rho a_{V_{3}}\left(\kappa_{10} \alpha_{t}+\kappa_{01} V_{t}\right) \\
C_{10}=0.5 \rho a_{V_{3}} \kappa_{00} V_{t}
\end{gathered}
$$


The system matrices $A_{i, j}, B_{i, j}$, and $H_{i, j}$ of Eq. (36) are

$$
\begin{aligned}
& H_{00}=\left[\begin{array}{cccc}
0 & 0 & h_{a V_{0}} & 0 \\
0 & 0 & h_{q V_{0}} & 0 \\
0 & 0 & h_{V V_{0}} & 0 \\
0 & 0 & 0 & 0
\end{array}\right], \quad H_{01}=\left[\begin{array}{cccc}
0 & 0 & h_{a V_{1}} & 0 \\
0 & 0 & h_{q V_{1}} & 0 \\
0 & 0 & h_{V V_{1}} & 0 \\
0 & 0 & 0 & 0
\end{array}\right], \\
& H_{02}=\left[\begin{array}{cccc}
0 & 0 & h_{a V_{2}} & 0 \\
k_{21} & 0 & h_{q V_{2}} & 0 \\
C_{21} & 0 & 0 & 0 \\
0 & 0 & 0 &
\end{array}\right], \quad H_{03}=\left[\begin{array}{cccc}
0 & 0 & 0 & 0 \\
0 & 0 & h_{q v_{3}} & 0 \\
0 & 0 & 0 & 0 \\
0 & 0 & 0 & 0
\end{array}\right] \\
& A_{00}=\left[\begin{array}{cccc}
0 & a_{\alpha_{1}} & 0.5 \rho a_{\alpha_{5}} \eta_{00} & 0 \\
0 & 0 & 0 & 0 \\
-a_{V_{2}} & 0 & 0 & a_{V_{2}} \\
0 & 1 & 0 & 0
\end{array}\right], \quad A_{01}=0.5 \rho\left[\begin{array}{cccc}
a_{\alpha_{5}} \eta_{10} & 0 & a_{\alpha_{5}} \eta_{01} & 0 \\
a_{q_{2}} & a_{q_{3}} & a_{q_{8}}+a_{q_{9}} \xi_{00} & 0 \\
0 & 0 & a_{V_{3}} \kappa_{00} & 0 \\
0 & 0 & 0 & 0
\end{array}\right] \text {, } \\
& A_{02}=0.5 \rho\left[\begin{array}{cccc}
0 & 0 & 0 & 0 \\
a_{q_{1}}+a_{q_{9}} \xi_{10} & 0 & a_{q_{9}} \xi_{01} & 0 \\
a_{V_{3}} \kappa_{10} & 0 & a_{V_{3}} \kappa_{01} & 0 \\
0 & 0 & 0 & 0
\end{array}\right], \quad A_{12}=0.5 \rho\left[\begin{array}{cccc}
0 & 0 & 0 & 0 \\
0.5 a_{q_{9}} \xi_{20} & 0 & 0 & 0 \\
a_{V_{3}} \kappa_{20} & 0 & 0 & 0 \\
0 & 0 & 0 & 0
\end{array}\right] \text {, } \\
& B_{00}=\left[\begin{array}{ccc}
0 & 0 & 0 \\
0 & 0 & a_{q_{7}} \\
0 & 0 & a_{V_{1}} \\
0 & 0 & 0
\end{array}\right], \quad B_{01}=\left[\begin{array}{ccc}
0.5 \rho a_{\alpha_{2}} \tau_{00} & 0 & 0 \\
0 & 0 & 0 \\
0 & 0 & 0 \\
0 & 0 & 0
\end{array}\right] \text {, } \\
& B_{02}=0.5 \rho\left[\begin{array}{ccc}
a_{\alpha_{2}} \tau_{01} & 0 & 0 \\
a_{q_{4}} \zeta_{00}+a_{q_{5}} & a_{q_{6}} & 0 \\
0 & 0 & 0 \\
0 & 0 & 0
\end{array}\right], \quad B_{03}=0.5 \rho\left[\begin{array}{ccc}
a_{\alpha_{2}} \tau_{02} & 0 & 0 \\
a_{q_{4}} \zeta_{01} & 0 & 0 \\
0 & 0 & 0 \\
0 & 0 & 0
\end{array}\right] \text {, } \\
& B_{04}=0.5 \rho\left[\begin{array}{ccc}
0 & 0 & 0 \\
a_{q_{4}} \zeta_{02} & 0 & 0 \\
0 & 0 & 0 \\
0 & 0 & 0
\end{array}\right], B_{r}=\left[\begin{array}{ccc}
0 & 0 & a_{\alpha_{3}} \\
0 & 0 & 0 \\
0 & 0 & 0 \\
0 & 0 & 0
\end{array}\right] \text {. }
\end{aligned}
$$




\section{Acknowledgments}

This research was supported by National Aeronautics and Space Administration under NASA Contract No. NAS1-02117. The first author thanks the technical monitor Dr. Gregory at the NASA Langley Research Center. Authors specially thank Dr. Andres Marcos and the research group of Dr. Balas at the University of Minnesota for the nonlinear simulation of the IFTC system.

\section{References}

[1] Belcastro, C., Khong, T., Shin, J-Y, Balas, G., Kwatny, H., and Chang, B., "Uncertainty Modeling for Robustness Analysis of Control Upset Prevention and Recovery Systems," in AIAA Guidance, Navigation and Control Conference, AIAA 2005-6427, AIAA, 2005.

[2] Shin, J-Y., N.E. Eva, and Belcastro, C., "Adaptive Linear Parameter Varying Control Synthesis for Actuator Failure," Journal of Guidance, Control, and Dynamics, Vol. 27, Sept.-Oct. 2004, pp. 787-794.

[3] Belcastro, C. and Chang, B-C., "Uncertainty Modeling for Robustness Analysis of Failure Detection and Accommodation Systems," in IEEE American Control Conference, Vol. 6, American Control Conference, 2002, pp. 4776-4782.

[4] Jordan, T., Langford, W., and Hill, J., "Airborne Subscale Transport Aircrat Research Testbed- Aircraft Model Development," in AIAA Guidance, Navigation and Control Conference, AIAA-2005-6432, AIAA, 2005.

[5] Bailey, R., Hostetler, R., Barnes K., Belcastro, Celeste, and Belcastro, Christine, "Experimental Validation: Subscale Aircraft Ground Facilities and Integrated Test Capability," in AIAA Guidance, Navigation and Control Conference, AIAA-2005-6433, 2005, AIAA.

[6] Foster, J., Cunningham, K., Fremaux, C., Shah, G., Stewart, E., and Wilborn, J., "Dynamic Modeling nd Simulation of Large Transport Airplaines in Upset Conditions," in AIAA Guidance, Navigation and Control Conference, AIAA-2005-5933, AIAA, 2005.

[7] Christopher, F., Andras, V., Bennani, S., and Selier, M., eds., Advanced Techniques for the Clearance of Flight Control Laws. Springer, 2002.

[8] Morton, B. and McAfoos, R., "A Mu-test for Robustness Analysis of Real-parameter Variation Problem," in Proceedings of the American Control Conference, Vol. 1, 1985, pp. 135-138.

[9] Morton, B., "New Application of mu to real-parameter Variations Problems," in IEEE Conference on Decision and Control, (Fort Lauderdale, FL), 1985.

[10] Belcastro, C., "On the Numerical Formulation of Parameteric Linear Fractional Transformation (LFT) Uncertainty Models for Multivariated Matrix Polynomial Problems," NASA, , November 1998. NASA TM-1998-206939.

[11] Belcastro, C., Lim, K., and Morelli, E., "Computer-Aided Uncertainty Modeling of Nonlinear Parameter-Dependent Systems, Part I: Thoretical Overview," in Proceeding of the Computer Aided Control System Design Conference, August 1999. 
[12] Belcastro, C., Lim, K., and Morelli, E., "Computer-Aided Uncertainty Modeling of Nonlinear Parameter-Dependent Systems, Part II:F-16 Example," in Proceeding of the Computer Aided Control System Design Conference, August 1999.

[13] Szaszi, I., Marcos, A., Balas, G., and Bokor, J., "Linear Parameter-Varying Detection Filter Design for a Boeing 747-100/200 Aircraft," Journal of Guidance, Control, and Dynamics, Vol. 28, No. 3, 2005, pp. $461-470$.

[14] Balas, G., Bokor, J., and Szabo, Z., "Failure Detection for LPV Systems-A Geometric Approach," in Proceedings of the American Control Conference, Vol. 6, 2002, pp. 4421-4426.

[15] Magni, J.-F., "Linear Fractional Representation Toolbox, Modeling, Order Reduction, Gain Scheduling," Systems Control and Flight Dynamics Department, ONERA, , Toulouse Cedex, France, 2004.

[16] Balas,G., Chiang, R., Packard, A., and Safonov M., Robust Control Toolbox. Natick, MA: The MathWorks, 2005.

[17] Marcos, A. and Balas, G., "Linear Parameter Varying Modeling of the Boeing 747-100/200 Longitudinal Motion," in AIAA Guidance, Navigation and Control Conference, AIAA-01-4347, American Institute of Aeronautics and Astronautics, (Montreal, Canada), Aug. 2001.

[18] Shin, J-Y., Worst-case Analysis and Linear Parameter Varying Control of Aerospace System. PhD thesis, Department of Aerospace Enginerring and Mechanics, University of Minnesota, 2000.

[19] Shin, J-Y., Balas, G.J., and Kaya, M.A., "Blending Methodology of Linear Parmeter Varying Control Synthesis of F-16 Aircraft System," Journal of Guidance, Control, and Dynamics, Vol. 25, No. 6, 2002, pp. 1040-1048.

[20] Shin, J-Y. and Belcastro, C., "Quasi-Linear Parameter Varying Representation over Non-trim Region," in AIAA Guidance, Navigation and Control Conference, AIAA-2004-5423, (Providence, Rhode Island), 2004, AIAA. 